\title{
Nanodimentional Thermometric NMR Sensors on the Basis of Double-Decker Paramagnetic Lantanide(III) Complexes with Porphyrins for Solution Temperature Measurements
}

\author{
Sergei P. Babailov \\ A.V. Nikolaev Institute of Inorganic Chemistry, Siberian Branch of Russian Academy of Sciences, 630090 Novosibirsk, Russia \\ E-mail: babajlov@niic.nsc.ru
}

\begin{abstract}
Earlier temperature dependencies of NMR spectra of asymmetric double-decker complexes of [LnH(oep)(tpp)], where Ln is Yb, Dy and Lu, tpp is tetraphenylporphyrin, and oep is octaethylporphyrin, have been analyzed by us [Babailov S.P., Coutsolelos A.G., Dikiy A., Spyroulias G.A., Eur. J. Inorg. Chem. 2001, 1, 303-306]. The NMR signal assignment has been done by $1 D$ NOE as well as on 2D NOESY, ROESY, TOECSY and COSY experiments. The kinetic and thermodynamic parameters for intramolecular phenyl ring rotations have been determined by dynamic NMR. The results obtained using pseudo-contact shifts and lanthanide-induced enhancement of longitudinal relaxation rates experienced by some protons of paramagnetic $[\mathrm{DyH}(\mathrm{oep})(\mathrm{tpp})]$ and $[\mathrm{YbH}(\mathrm{oep})(\mathrm{tpp})]$ revealed that the structure of the complexes are very similar, with only slight decrease in distance between the planes of porphyrin ligands upon increase in the atomic number of the lanthanide ions. This result has been interpreted as being due to lanthanide contraction. Nevertheless, paramagnetic properties of these complexes were investigated insufficiently. In the paper the ${ }^{1} H$ NMR method is used to study the features of paramagnetic properties of complexes [DyH(oep)(tpp)]. The temperature dependencies of the lanthanide-induced shifts in NMR spectra of asymmetric double-decker complexes of [DyH(oep) (tpp)] are analyzed. The obtained results indicate that complexes [DyH(oep)(tpp)] are promising compounds as in situ NMR thermosensors for temperature determination in solution.
\end{abstract}

Keywords: Lantanide-induced shifts, paramagnetic susceptibility, nuclear magnetic resonance, thermometric NMR sensors, lantanide complexes with porphyrins.

\section{Наноразмерные термометрические ЯМР-сенсоры на основе Авухпалубных парамагнитных комплексов лантанидов(III) с порфиринами Аля определения температуры в растворах}

\author{
С. П. Бабайлов
}

Институт неорганической химии им. А.В. Николаева СО РАН, 630090 Новосибирск, Россия

E-mail: babajlov@niic.nsc.ru

\begin{abstract}
Ранее нами с помощьью 1D и 2D ЯМР спектроскопии были исследованы парамагнитные двухпалубные комплексы лантанидов(III) с порфиринами [LnH(oеp)(tpp)] и обнаружено увеличение энтальпии активации $\left(\Delta \mathrm{H}^{*}\right)$ вращения фенильных колец анионов порфиринов с ростом атомного номера катиона Ln, которое обусловлено эффектом лантанидного сжатия, где оер-октаэтилпорфирин, tрp-тетрафенилпорфирин (см. [Babailov S.P., Coutsolelos A.G., Dikiy A., Spyroulias G.A. Eur. J. Inorg. Chem. 2001, 1, 303-306]). В ходе исследований молекулярной динамики проводился анализ температурного изменения спектров ЯМР, однако результаты исследования температурного изменения лантанид-индуцированных сдвигов (ЛИС) не были детально проанализированы и опубликованьл. В связи с этим в настоящей работе нами исследованы температурные изменения ЛИС для атомов водорода различных групп лигандов для случая комплексов [LnH(oep)(tpp)], где Ln=Dy и Lu. Полученные результаты, a также найденная высокая термодинамическая и кинетическая стабильность в сочетании с мальми размерами молекул комплексов [LnH(оер)(tpp)] в растворах создают предпосылки использования этих соединений в качестве термометрических ЯМР-сенсоров непосредственно в реакиионных средах для іп situ определения температуры в растворе и изучения процессов, протекающих с выделением или поглощением тепла.
\end{abstract}

Ключевые слова: Лантанид-индуцированные сдвиги в спектрах ЯМР, парамагнитная восприимчивость, ядерный магнитный резонанс, термометрический ЯМР-сенсор, комплексы лантанидов с порфиринами. 


\section{Введение}

Информация о молекулярной динамике, строении и парамагнитных свойствах координационных соединений лантанидов (Ln) в растворах лежит в основе существующих технологий получения препаратов для фотодинамической терапии, контрастных реагентов для медицинской магниторезонансной томографии (ММРТ) и сенсоров для биологии и медицины. ${ }^{[1-5]}$ Методы ядерного магнитного резонанса (ЯМР) хорошо себя зарекомендовали для получения такой информации в растворах. ${ }^{[3]} \mathrm{B}$ парамагнитных комплексах Ln за счет лантанид-индуцированных сдвигов (ЛИС) в спектрах ЯМР на несколько порядков расширяется диапазон доступных для измерения констант скоростей химического обмена (ХO) по сравнению с диапазоном констант скоростей в родственных диамагнитных соединениях. ${ }^{[3]}$ Как установлено на широком классе соединений, использование динамического ЯМР (ДЯМР) для определения внутримолекулярной динамики лигандов парамагнитных комплексов Ln можно рассматривать как новый методический прием изучения кинетики процессов ХО, протекающих в лигандах, со своей характерной областью применения. ${ }^{[3]}$ Этот методический подход ранее апробирован на ряде примеров ДЯМР-исследования кинетики межмолекулярной и конформационной динамики краун-эфиров, порфиринов, а также и EDTA, координированных с Ln в растворах. ${ }^{[3-5]}$ В частности ранее нами при исследовании методом ДЯМР двухпалубных парамагнитных комплексов лантанидов(III) с порфиринами [LnH(oep)(tpp)] было обнаружено увеличение энтальпии активации $\left(\Delta \mathrm{H}^{\ddagger}\right)$ вращения фенильных колец анионов порфиринов с ростом атомного номера катиона Ln, которое обусловлено эффектом лантанидного сжатия, где оер - октаэтилпорфирин, tрp - тетрафенилпорфирин (Рисунок 1). ${ }^{[3,6]}$

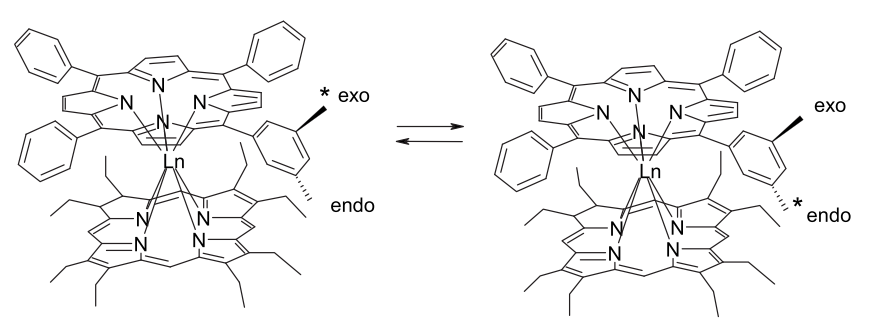

Рисунок 1. Схема внутримолекулярной динамики и обозначение атомов водорода в комплексах лантанидов(III) с порфиринами [LnH(oep)(tpp)], где оер - октаэтилпорфирин, tpp - тетрафенилпорфирин.

Отнесение сигналов в ${ }^{1} \mathrm{H}$ ЯМР спектрах комплексов [LnH(oep)(tpp)] в этом исследовании было осуществлено с применением 1D NOE, а также 2D NOESY, ROESY, TOECSY и COSY.[ ${ }^{6]}$ Необходимо отметить, что помимо результатов исследования динамики на незначительное изменение межплоскостного расстояния между анионами порфиринов (в зависимости от катиона Ln) в исследованных двухпалубных комплексах также указывал проведенный комбинированный анализ ЛИС и парамагнитных увеличений скоростей спин-решеточной релаксации на ядрах лигандов. ${ }^{[6]}$ Кроме того был про- веден детальный анализ значений ЛИС и парамагнитных увеличений скоростей спин-решеточной релаксации. Конкретно в диамагнитных комплексах [LuH(oep)(tpp)] были отнесены следующие сигналы с химическими сдвигами ( $\delta$, м.д.) относительно ТМС: 9.1 (мезо), 4.1 (эндо- $\mathrm{CH}_{2}$ ), 3.7 (экзо- $\mathrm{CH}_{2}$ ), 9.4 (эндо-орто), 6.4 (экзоорто), 7.6 (пара), 8.1 (эндо-мета), 7.2 (экзо-мета), 1.3 $\left(\mathrm{CH}_{3}\right), 8.1$ (пиррольный). Парамагнитные сдвиги в ${ }^{1} \mathrm{H}$ ЯМР спектрах парамагнитных комплексов [DyH(oеp) (tpp)] были определены при температуре 298 K $(\Delta \delta$, м.д.) относительно диамагнитных комплексов [LuH(oep) (tpp)]: -21.7 (мезо), -6.6 (эндо- $\left.\mathrm{CH}_{2}\right),-8.2$ (экзо- $\left.\mathrm{CH}_{2}\right),-59.3$ (эндо-орто), 16.2 (экзо-орто), -9.6 (пара), -21.9 (эндомета), -3.4 (экзо-мета), -3.8 ( $\left.\mathrm{CH}_{3}\right),-69.5$ (пиррольный). [6] Дополнительную информацию об особенностях строения и физико-химических свойствах в кристаллах и растворах двухпалубных комплексов лантанидов(III) с порфиринами можно получить из литературы. ${ }^{[7-10]}$ При исследовании молекулярной динамики нами учитывалось температурное изменение парамагнитных ЛИС ${ }^{[6]}$ (в рамках методик, детально обсужденных в обзоре ${ }^{[3]}$ ), однако результаты анализа температурного изменения ЛИС в двухпалубных парамагнитных комплексах лантанидов(III) с порфиринами не были опубликованы. Вместе с тем, этот анализ может представлять самостоятельный интерес. Дело в том, что одним из практических применений комплексов Ln является использование найденной существенной зависимости парамагнитных ЛИС от температуры для определения методом ЯМР температуры в жидких средах. ${ }^{[4,5]}$ В связи с этим, комплексы Ln можно рассматривать как ЯМР-термосенсоры для определения температуры жидких сред и перспективными термочувствительными реагентами для ММРТ. [4,5] Причем одними из наиболее интересных являются комплексы Dy, так как именно в комплексах Dy наблюдаются наиболее существенные псевдоконтактные вклады в ЛИС по сравнению с другими лантанидами. ${ }^{[3]}$

В связи с этим в настоящей работе нами исследованы температурные изменения ЛИС для атомов водорода различных групп лигандов для случая комплексов [LnH(oep)(tpp)], где Ln= Dу и Lu.

\section{Экспериментальная часть}

Спектры ЯМР снимались на серийных импульсных спектрометрах Bruker MSL-300 и Bruker Avance-III-500 (рабочие частоты ${ }^{1} \mathrm{H}$ ЯМР соответственно 300.038 МГц и 500.13 МГц). Стабилизация постоянного магнитного поля осуществлялась с помощью комплектных блоков использованных спектрометров по сигналам ЯМР на ядрах дейтерия от специально применяемых дейтерий-обогащенных растворителей. Концентрация комплексов при исследовании температурных зависимостей составляла $5 \cdot 10^{-3} \mathrm{M}$.

Температура в исследуемых образцах регулировалась с помощью комплектных температурных блоков с погрешностью \pm 0.3 К. Погрешность и стабильность (которая была \pm 0.3 К) функционирования температурных блоков контролировалась с помощью комплектных стандартных образцов с известной графической зависимостью химических сдвигов в спектре ${ }^{1} \mathrm{H}$ ЯМР от температуры.

Синтез комплексов [ $\mathrm{LnH}(\mathrm{oep})(\mathrm{tpp})]$ проводился аналогично работе. ${ }^{[6]}$ 


\section{Результаты и обсуждение}

Как обнаружено экспериментально, наблюдается существенная зависимость ЛИС на ядрах ${ }^{1} \mathrm{H}$ различных групп, а также разности ЛИС, соответствующих различным группам анионов лигандов, от обратного значения абсолютной температуры (1/T). Исследованные в диапазоне температур от 225 до 330 К зависимости ЛИС хорошо описываются линейной аппроксимацией от обратного значения абсолютной температуры $\delta_{1}-\delta_{2}=\mathrm{A}+\mathrm{B} / \mathrm{T}$, где параметры А и В рассчитаны с помощью линейной регрессии (Рисунки 2 и 3 ).

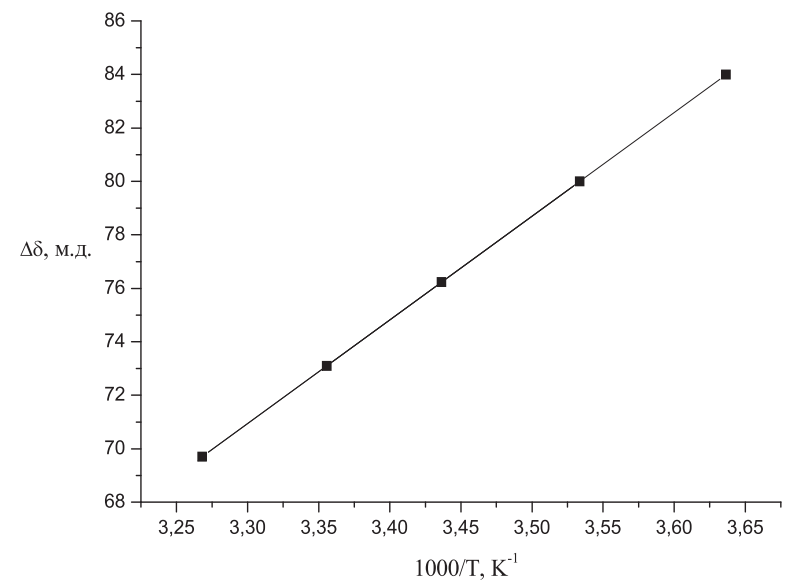

Рисунок 2. Зависимость разности химических сдвигов между орто-экзо и орто-эндо атомами фенильного кольца аниона порфирина в комплексах [DyH(oep)(tpp)] от обратной величины температуры (в растворителе $\mathrm{CDCl}_{3}$ ).

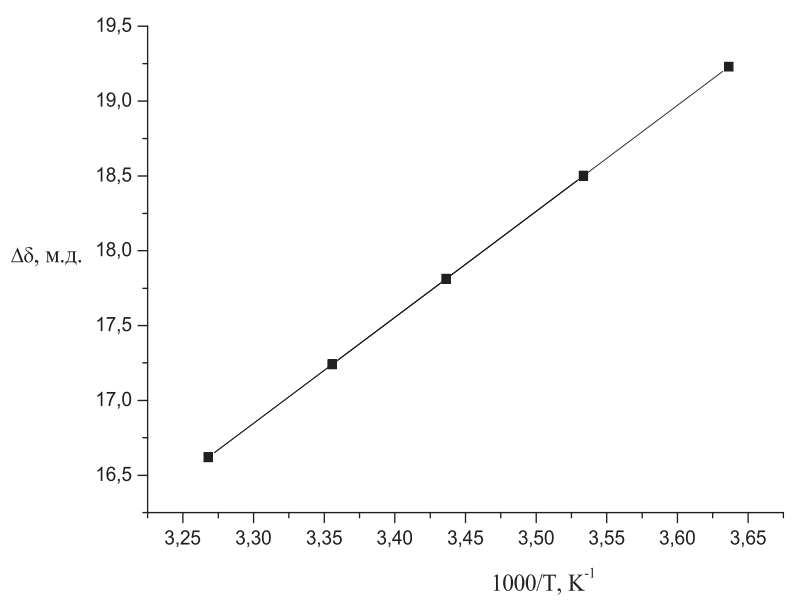

Рисунок 3. Зависимость разности химических сдвигов между мета-экзо и мета-эндо атомами фенильного кольца аниона порфирина в комплексах [DyH(oep)(tpp)] от обратной величины температуры (в растворителе $\mathrm{CDCl}_{3}$ ).

Найденные значения параметров А и В для парамагнитных комплексов Dу соответственно равны: -57(2) м.д. и $38800(500)$ К·м.Д. (для разности между сигналами атомов водорода орто-экзо и орто-эндо); -6.5(9) м.д. и 7080(100) К·м.д. (для разности между сигналами атомов водорода мета-экзо и мета-эндо); -42.2(8) м.д. и -32600(600) К·м.д. (для пиррольных атомов водорода, рассчитанных относительно диамагнитных комплексов $\mathrm{Lu}$. Аналогичная температурная зависимость была получена и для пиррольных атомов водорода анионов порфиринов в комплексах [DyH(oеp)(tpp)] (Рисунок 4).

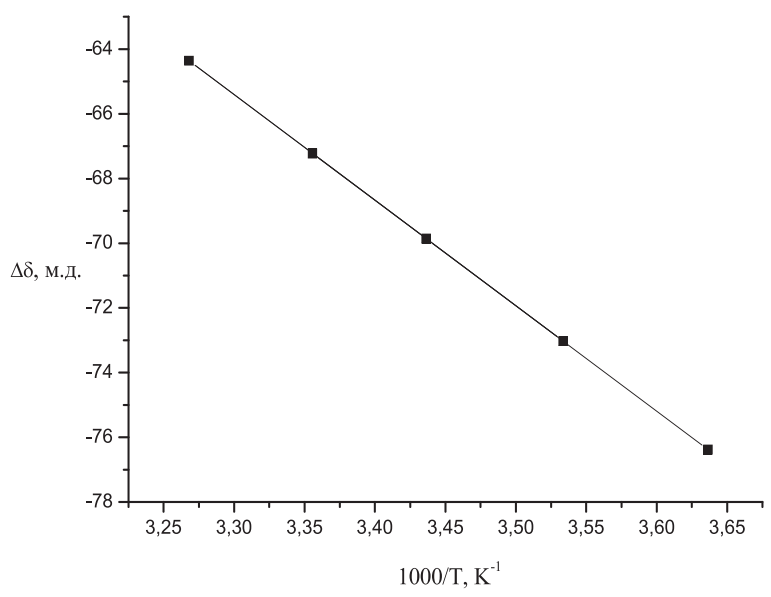

Рисунок 4. Зависимость парамагнитных химических сдвигов пиррольных атомов фенильного кольца аниона порфирина в комплексах [DyH(oep)(tpp)] от обратной величины температуры (в растворителе $\mathrm{CDCl}_{3}$, относительно диамагнитных комплексов [ $\mathrm{LuH}(\mathrm{oep})(\mathrm{tpp})])$.

Этот результат согласуется с результатами исследования температурной зависимости в других кинетически стабильных комплексах $\operatorname{Ln}$, например, в $[\operatorname{Ln}(\mathrm{EDTA})]$ и $\left[\operatorname{Ln}\left(18-\text { краун-6) } \mathrm{L}_{\mathrm{i}} \mathrm{L}_{(2-\mathrm{i})}\right]^{+}\left[\mathrm{LnL}_{\mathrm{j}} \mathrm{L}_{(4-\mathrm{j})}\right]^{-}\right.$, где $\mathrm{L}^{\prime}$ - гептафтордиметилоктандионато или гексафторацетилацетонато анионы, причем $\mathrm{i}=0,1,2, \mathrm{a} j=0,1,2,3,4$. $^{[3-6]}$

Известно, что Ферми-контактные вклады ЛИС пропорциональны $1 / \mathrm{T}$, а в теоретической работе Б. Блини ${ }^{[11]}$ прогнозировалось, что псевдоконтактные сдвиги в парамагнитных комплексах Ln должны быть пропорциональны $1 / \mathrm{T}^{2}$. Что касается экспериментальных исследований, то некоторые из них подтверждали зависимость $1 / \mathrm{T}^{2}$ (однако в этих работах не было тщательного контроля термодинамической и кинетической стабильности исследуемых комплексов), а некоторые не подтверждали или сводились к следующей зависимости псевдоконтактных вкладов в ЛИС::[12]

$$
\Delta \delta_{\mathrm{PC}}=\mathrm{A}+\mathrm{B} / \mathrm{T}
$$

Нами поддерживается точка зрения, высказанная в работе ${ }^{[12]}$ согласно которой температурная зависимость ЛИС существенно зависит от термов более высоких порядков (т.е. $\left.1 / \mathrm{T}^{\mathrm{n}}\right)$, однако для практических целей в рабочих диапазонах от 200 до 350 К экспериментальная зависимость хорошо описывается линейной аппроксимацией (1).

Как видно из найденных значений параметров А и В в комплексах [DyH(oep)(tpp)], имеется существенная экспериментальная зависимость разности ЛИС от температуры, что может быть использовано для практического применения этих комплексов в качестве ЯМРтермосенсоров для определения температуры растворов. Необходимо отметить, что для контроля температуры в образце могут использоваться ЛИС индивидуальных 
сигналов групп (взятые относительно соответствующих сигналов диамагнитных комплексов Lu). Однако, меньшая погрешность измерения температуры достигается при использовании разности ЛИС, соответствующих неэквивалентным группам лигандов парамагнитного комплекса. Согласно проведенному анализу молекулярного строения ${ }^{[3,6]}$ в растворе, минимальный размер молекул комплексов в направлении, перпендикулярном плоскости анионов порфиринов, составляет $\approx 1.1 \mathrm{нм.}$ Экспериментальные результаты свидетельствуют о том, что наиболее подходящими наноразмерными зондами для определения температуры (или термометрическими ЯМР-сенсорами) в растворе $\mathrm{CDCl}_{3}$ оказались комплексы Dy.

Исследованием концентрационной зависимости значений константы скорости химического обмена, представленного на Рисунке 1, установлено отсутствие зависимости значений от исходной концентрации [DyH(oep)(tpp)] в растворе $\mathrm{CDCl}_{3}$ (диапазон измерения концентрационной зависимости от $10^{-3}$ до $1.1 \cdot 10^{-2} \mathrm{M}$ ), что свидетельствует о первом или псевдопервом порядке реакции. Исследование в этом же концентрационном диапазоне значений химических сдвигов комплексов [DyH(oep)(tpp)] свидетельствует, что они практически не зависят от концентрации (это указывает на кинетическую стабильность исследуемых комплексов в растворах).

Высокая термодинамическая и кинетическая стабильность в сочетании с малыми размерами молекул [LnH(oep)(tpp)] в растворах создают предпосылки использования этих соединений в качестве термометрических ЯМР-сенсоров непосредственно в реакционных средах (для in situ контроля температуры при изучении обратимых и необратимых фотохимических реакций, ${ }^{[13]}$ процессов, протекающих с выделением или поглощением тепла), а также в магниторезонансной томографии для трехмерного картографирования распределения температуры в жидких средах и в перспективе в некоторых пористых соединениях. Так, например, в ходе in situ исследования какой-либо фотохимической реакций в ампуле для спектроскопии ЯМР с диаметром 5 мм при лазерном световом воздействии сверху, в условиях, когда оптическая плотность образца $(D)$ близка к единице, вполне возможен неравномерный нагрев исследуемого образца (т.к. свет поглощается в соответствии с законом Ламберта-Бера, а КПД фотохимических процессов как правило меньше $100 \%) .{ }^{[13]}$ Этот фотоиндуцированный градиент температуры в образце вполне возможно контролировать с помощью предлагаемых термометрических ЯМР-сенсоров.

\section{Заключение}

Высокая термодинамическая и кинетическая стабильность в сочетании с малыми размерами молекул комплексов [LnH(oep)(tpp)]) в растворах создают предпосылки использования этих соединений в качестве термометрических наноразмерных ЯМР-сенсоров непосредственно в реакционных средах (для in situ изучения процессов, протекающих с выделением или поглощением тепла), а также для контроля температуры при изучении обратимых и необратимых фотохимических реакций.

Благодарность. Работа выполнена при частичной финансовой поддержке гранта №67 Президиума СО РАН.

\section{Список литературы}

\section{References}

1. Parker D., Dickins R.S., Puschmann H., Crossland C., Howard J.A.K. Chem. Rev. 2002, 102, 1977-2010.

2. Piguet C., Geraldes C.F. Handbook on the Physics and Chemistry of Rare Earths (Gschneidner Jr. K.A., Bünzli J.-C. G., Pecharsky V.K., Eds.). Amsterdam: Elsevier Science, 2003; Vol. 33, p. 353-463.

3. Babailov S.P. Prog. Nucl. Magn. Reson. Spectrosc. 2008, 52, 1-21.

4. Babailov S.P. Russ. Chem. Bull. 2008, 57, 1317-1318 [Izv. Ross. Akad. Nauk, Ser. Khim. 2008, 6, 1292-1294 (in Russ.)].

5. Babailov S.P., Kokovkin V.V., Stabnikov P.A. Rus. J. Struct. Chem. 2010, 51, 652-656 [Zh. Struktur. Khimii 2010, 51, 682686 (in Russ.)].

6. Babailov S.P., Coutsolelos A.G., Dikiy A., Spyroulias G.A. Eur. J. Inorg. Chem. 2001, 1, 303-306.

7. Buchler J.W., Kapellmann H.G., Knoff M., Lay K.-L., Pfeifer S. Z. Naturforsch 1983, 38B, 1339-1345 (in Germ.).

8. Spyroulias G.A., Raptopoulou C., de Montauzon D., Mari A., Poilblanc R., Terzis A., Coutsolelos A.G. Inorg. Chem. 1999, 38, 1683-1696.

9. Spyroulias G.A., Coutsolelos A.G. Inorg. Chem. 1996, 35, 1382-1385.

10. Spyroulias G.A., Coutsolelos A.G., Raptopoulou C., Terzis A. Inorg. Chem. 1995, 34, 2476-2479.

11. Bleaney B. J. Magn. Reson. 1972, 25, 91-100.

12. De Boer J.W.M., Sakkers P.J.D., Hilber C.W., De Boer M. J. Magn. Reson. 1977, 25, 455-476.

13. Babailov S.P. Prog. Nucl. Magn. Reson. Spectrosc. 2009, 54, 183-194. 